\title{
Bilirubin Binding in the Plasma of Newborns: Critical Evaluation of a Fluorescence Quenching Method and Comparison to the Peroxidase Method
}

\author{
CHARLES B. BERDE, ${ }^{(50)}$ WILLIAM E. BENITZ, L. FRASER RASMUSSEN, JOHN A. KERNER, ${ }^{(54)}$ \\ JOHN D. JOHNSON, ${ }^{(51)}$ AND RICHARD P. WENNBERG \\ Department of Pediatrics, Stanford University, School of Medicine, Stanford, California, [C.B.B., W.E.B., J.A.K., \\ J.D.J.J and Department of Pediatrics, University of California School of Medicine, Davis, California, USA
}

[L.F.R., R.P.W.]

\section{Summary}

Bilirubin binding affinities and capacities and apparent unbound ("free") bilirubin levels were determined in serum samples from 47 high-risk newborns, in 22 samples of cord serum, and in serum samples from 15 Greek children with marked hyperbilirubinemia, by both fluorescence quenching and peroxidase methods. The free fatty acid:albumin molar ratio was also determined for serum samples from high-risk newborns. In vitro and in vivo measurements suggest that free fatty acids are rarely present at levels that produce significant displacement of bilirubin, which is in agreement with previous studies. The two bilirubin binding assays showed only fair correlation with sizable discrepancies for many specimens. Technical difficulties inherent in the fluorescence quenching method and possible sources of error are discussed. Our observations suggest that routine application of these two assays as the primary criterion for therapeutic intervention (e.g., exchange transfusion) is premature.

\section{Abbreviations}

(c), unbound bilirubin concentration

FA/SA, fatty acid/serum albumin molar ratio

HSA, human serum albumin

$K_{d}$, dissociation constant

$\mathrm{n}$, total bilirubin binding capacity

RBBC, reserve bilirubin binding capacity

$\nu$, bound bilirubin/total albumin molar ratio

Since the classic studies of Silverman et al. (39), Harris et al. (20), and Odell et al. (30) in the 1950s, the prevailing view has been that reduction of the binding affinity or capacity of albumin for bilirubin increases the quantity of bilirubin available for adsorption to brain tissues, which in turn increases the risk of kernicterus. Many drugs and endogenous ligands which bind to albumin, such as free fatty acids, have been reported to reduce bilirubin binding affinities or capacities $(3,9,10,32,36,40,41$, $42,48,49)$. A variety of methods have been proposed to assess bilirubin binding $(2,4,7,11,13,17,19,22,23,25,28,31,35$, 48). Most of these methods attempt to measure binding capacity or reserve binding capacity but some also attempt to measure binding affinity or unbound bilirubin. As discussed in detail by Brodersen (8), most clinically important displacers appear to reduce bilirubin binding primarily via changes in apparent binding affinity rather than via changes in binding capacity; thus, measurement of the reserve binding capacity alone may be a relatively insensitive index of bilirubin displacement.

In addition, factors altering bilirubin binding to tissues or the susceptibility of tissues to bilirubin toxicity cannot be assessed by methods that measure only the binding of bilirubin to albumin. One new method, the fluorescence enhancement method $(11,13)$, measures both albumin bound and non-albumin bound (largely red cell bound) bilirubin. The latter is affected by both the bilirubin binding capacity and affinity of albumin, and by the tissue binding affinity. This new method may reflect the risk of tissue toxicity better than methods which measure only the binding of bilirubin to albumin.

The peroxidase and fluorescence quenching methods used in this study attempt to establish apparent binding constants and capacities and unbound bilirubin levels. We address here the empirical question of whether or not these two methods agree in serum samples from high-risk newborns, cord serum from healthy newborns, and samples from newborns with severe hyperbilirubinemia. In an attempt to identify some of the sources of variation in measurements, we compared binding data with fatty acid:albumin molar ratios in plasma (6).

\section{MATERIALS AND METHODS}

Biochemicals and reagents. Bilirubin (Sigma) was recrystallized from chloroform-methanol according to the procedure of Ostrow et al. (33). Purity was judged to be adequate by virtue of spectrophotometric extinction coefficients in agreement with reported values (14). Solutions of bilirubin were prepared fresh for each series of assays by dissolving the bilirubin in a small amount of $0.05 \mathrm{~N} \mathrm{NaOH}$ and then diluting the solution in distilled water. All operations involving bilirubin were performed in a darkened room in dim red light. Human serum albumin (Miles) was treated by the procedure of Chen (15) to remove fatty acids, and monomer was isolated by Sephadex G-150 gel filtration as described by Morrisett et al. (29). Monomer and dimer had similar binding properties by both fluorescence quenching and peroxidase methods. Electrophoresis (18) indicated greater than $98 \%$ purity as judged by Coomassie blue staining and densitometry. Linolenic acid (Sigma) showed a single spot on thin layer chromatography and greater than $98 \%$ purity by gas-liquid chromatography of the methyl ester. Solutions were used for no longer than $4 \mathrm{~h}$ after preparation.

Spectroscopic methods. Albumin determinations in plasma samples were performed by the bromcresol green method, as described by Doumas et al. (16). Total bilirubin was measured 
by both direct absorbance (21) and diazo (27) methods. When a discrepancy was observed, the diazo method was chosen as more specific, especially in the presence of hemolysis or lipemia. Peroxidase measurements were performed as reported previously (48). Fluorescence quenching was measured as described by Levine (25), with the following modifications (5): a Perkin-Elmer quartz microcuvette $(0.4 \mathrm{~cm}$ optical path) was used to reduce inner filter effects and to permit measurements on more concentrated samples. To minimize errors resulting from dilution, test solutions (dilutions of plasma) were prepared in volumes which were large $(7-30 \mathrm{ml})$ relative to the volumes of bilirubin additions (5-10 $\mu \mathrm{l})$; samples removed from the test solution for fluorescence assay (cuvette volume $0.3 \mathrm{ml}$ ) were returned to the test solution before titration was continued; fluorescence intensities were corrected for dilution and inner filter effects. As described by Levine (25), the amount of unbound bilirubin at each level of added bilirubin was determined by extrapolation of the initial slope of a plot of relative fluorescence intensity versus the bilirubin:albumin molar ratio and noting the deviation of the experimental curve from this line. Such a method can overestimate the binding affinity if the initial slope is derived at too great a dilution (34); therefore, we used a 50-100-fold dilution of serum to obtain the initial slope, and greater dilutions (350-700-fold) to obtain the experimental curve. This necessity for measurements at different dilutions is an unfortunate feature of this method. In our experience, it is necessary to use large numbers of small additions in the titration in order to obtain reproducible results. We used eight aliquots (each increasing the biliru. bin:albumin molar ratio by $0.025-0.05$ ) to determine the initial slope and six to fifteen subsequent additions to determine the binding curve. Fluorescence measurements were made using a Perkin-Elmer Model MPF 2-A fluorimeter equipped with excitation and emission monochromaters and a circulating water bath for temperature control. The use of two monochromaters is necessary to eliminate scattering artifacts. When determined in this way on a series of identical quick-frozen samples, the inday coefficients of variation $(n=20)$ for affinities and capacities were 0.08 and 0.14 , respectively, and the between-day coefficients of variation were 0.14 and 0.06 , respectively. In our opinion, it is necessary to use rather extreme measures in order to obtain adequate reproducibility with this method. Fatty acid:albumin molar ratios were determined using the parinaric acid absorption method, as described previously (6).

Data analysis. We define $\nu$ as the molar ratio of bound bilirubin to total albumin and (c) as the unbound bilirubin concentration. The method of Scatchard (38) derives the association constant (binding constant) as the negative slope of a plot of $\nu /(\mathrm{c})$ versus $\nu$ whereas the binding capacity, $\mathrm{n}$, is defined as the $x$-intercept of a linear extrapolation of the segment of this curve having values of $\nu$ less than 1 . The reserve bilirubin binding capacity $(\mathrm{RBBC})$ is defined as $n-\nu$. Note that by these definitions $\mathrm{n}$ and $\mathrm{RBBC}$ are expressed not as bilirubin concentrations, but as molar ratios of bilirubin to albumin. The reader should exercise caution because not all previous treatments have defined $\mathrm{RBBC}$ in this way. Plots of $\nu /(\mathrm{c})$ versus $\nu$ are generally quite linear up to values of $\nu$ around 0.8 , and linear least squares was used to estimate slope and intercept. Scatchard analysis used in this way exaggerates the importance of data derived at low levels of unbound bilirubin and ignores second-site binding. This method was chosen because: 1) it is the method used by the originators of both methods, 2) it is convenient, and 3) binding capacities and reserve binding capacities have been important in previous discussions of bilirubin binding, and these quantities are less naturally derived from more general multiple equilibrium models. We report dissociation constants rather than binding or association constants (the reciprocals of dissociation constants), because these are directly, rather than inversely, proportional to the unbound bilirubin concentration, the presumed parameter of clinical interest.

Patient selection and sample collection. High-risk neonates were selected for study on the basis of having one or more of the following attributes: 1) total serum bilirubin level greater than $10 \mathrm{mg} / \mathrm{dl}$, 2) birth weight less than $1200 \mathrm{~g}, 3)$ receiving intravenous lipid supplements or furosemide, or 4) having evidence of hemolytic disease by Coomb's test. Blood sampling at Stanford University Medical Center followed parental informed consent according to a protocol approved by the Medical Committee for Use of Human Subjects in Research. At the University of California Medical Center, Davis, measurement of unbound bilirubin is a standard procedure for assessing risk of bilirubin encephalopathy in neonates, and samples were obtained as a part of routine patient monitoring. Plasma samples were obtained via arterial line, venipuncture, or heel stick. In each case, blood was drawn only in conjunction with other tests necessary for patient management. Blood was collected in heparinized vessels, chilled to $0^{\circ} \mathrm{C}$, removed from light, and centrifuged to obtain plasma. Plasma samples were sealed and stored in the dark at $0^{\circ} \mathrm{C}$. Experiments were arranged so that measurements of bilirubin binding by both methods were performed on split samples at approximately the same time after the blood was drawn, whenever possible. Repeated measurements by both methods indicate that dissociation constants and binding capacities are unchanged to within $10 \%$ for samples stored this way for up to $2 \mathrm{wk}$. It is undeniable that storage of samples containing bilirubin is problematic; we have attempted to make all such sources of error at least comparable for the two methods of measurement. Cord serum was handled in the same manner as the high-risk neonate serum. Samples from Greek infants with marked hyperbilirubinemia were obtained and handled as described previously (48).

\section{RESULTS}

As shown in Figure 1, the values of $K_{d}, n, R B B C$, and unbound bilirubin determined by these two methods are significantly correlated; however, there is substantial dispersion of the individual values. Bilirubin binding parameters were measured by both methods using 22 samples of cord blood from healthy full-term infants. Again, Table 1 shows only fair correlation between the two methods. There were no significant differences between the high-risk newborns and the healthy newborns in the mean values of $K_{d}$ or $n$ by either method (Table 2). The differences in unbound bilirubin and RBBC are purely a consequence of the differences in $\nu$ between the two groups.

Neither method showed a significant dependence of $K_{d}$ or $n$ on birth weight or estimated gestational age (Table 3). There was a slight, but significant, increase in unbound bilirubin at lower birth weights and gestational age by the peroxidase method, but not by the fluorescence quenching method (Table 3 ). In this case, the dependence of unbound bilirubin on birth weight and gestational age is not a trivial consequence of a trend in $\nu$ or total bilirubin because these showed no dependence on birth weight or gestational age (Table 3 ).

Newborns receiving phototherapy showed no systematic differences from the newborns not receiving phototherapy for any binding parameter. Only two infants had levels of direct bilirubin greater than $1.5 \mathrm{mg} / \mathrm{dl}$ and there was no suggestion of a difference between the two methods for those samples.

Only seven of our serum samples had values of $\nu$ greater than 0.6 . To compare the use of the two methods for serum samples having high values of $\nu$, bilirubin binding measurements were attempted on serum samples from 15 Greek children with marked hyperbilirubinemia (all had values of $\nu$ greater than 0.6 ). For all of these samples, the initial segments of the plot of fluorescence intensity versus $\nu$ were curved and, thus, it was impossible to obtain any quantitative information by the fluorescence quenching method. With the peroxidase method, it was possible to estimate the level of unbound bilirubin without a titration, and all of these samples had high levels of unbound bilirubin.

It has been suggested previously that an unbound bilirubin level greater than $20 \mathrm{nM}\left(2 \times 10^{-8} \mathrm{M}\right)$ by the peroxidase method should be considered potentially risky and should influence the 

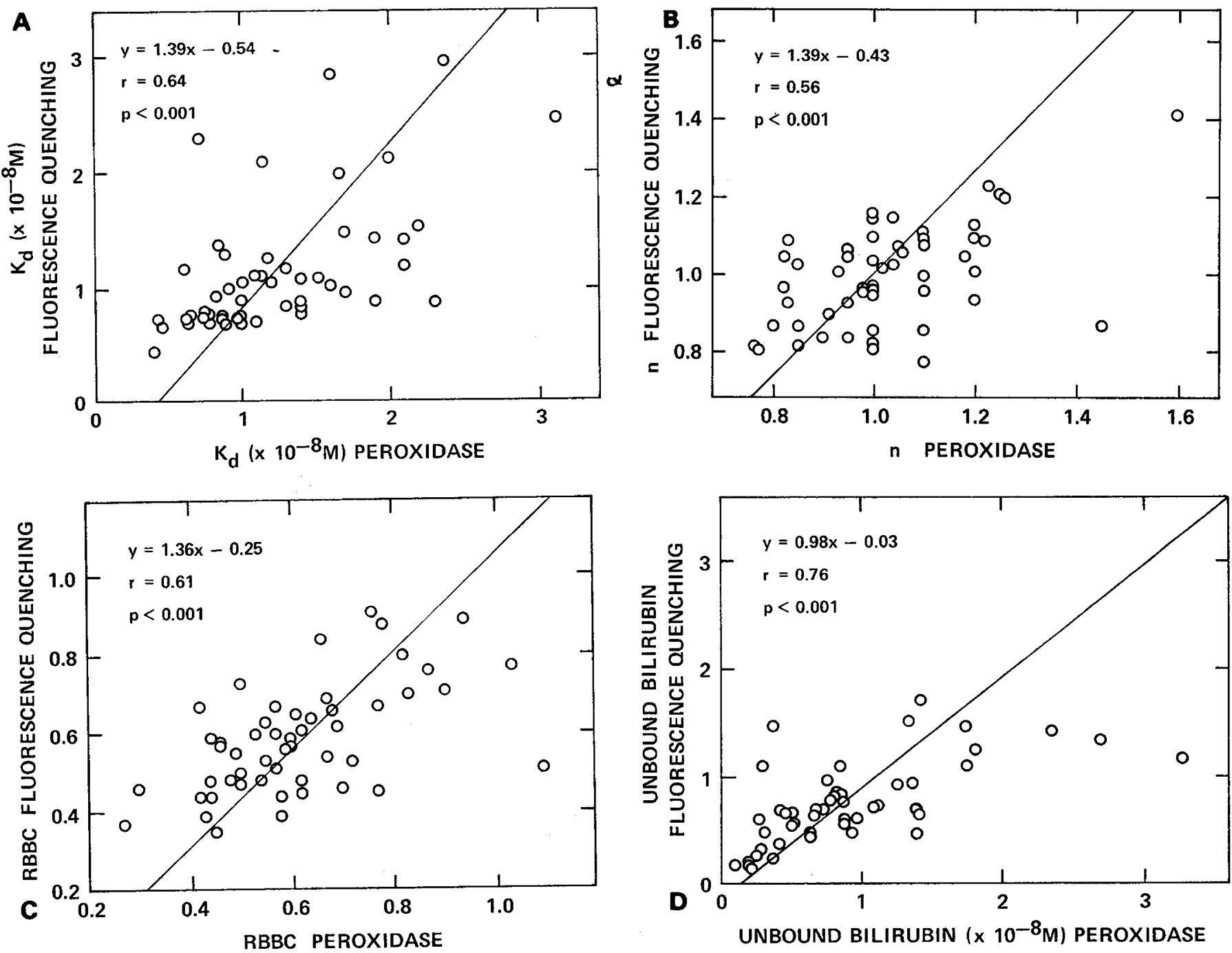

Fig. 1. Comparison of binding parameters for high-risk newborns between fluorescence quenching and peroxidase methods. Panels A-D show measurement of $\mathrm{K}_{\mathrm{d}}, \mathrm{n}$, reserve bilirubin binding capacity, and unbound bilirubin as described in "Materials and Methods." In each panel, $\mathrm{y}=\mathrm{mx}$ $+\mathrm{b}$ is the linear least squares line, $r$ is the correlation coefficient, and $P$ is the significance level. (Some circles represent more than one data point.)

Table 1. Comparison of fluorescence quenching and peroxidase measurement of binding parameters for cord blood samples from healthy term newborns*

\begin{tabular}{lcccc}
\hline Parameter & Slope & Intercept & $r$ & $P$ \\
\hline $\mathrm{n}$ & 0.39 & 0.66 & 0.58 & 0.002 \\
$\mathrm{RBBC} \dagger$ & 0.44 & 0.58 & 0.63 & $<0.001$ \\
$\mathrm{~K}_{\mathrm{d}}$ & 0.34 & 0.56 & 0.47 & 0.01 \\
$\quad \begin{array}{l}\text { Unbound bil- } \\
\quad \text { irubin }\end{array}$ & 0.65 & 0.01 & 0.86 & $<0.001$ \\
\hline
\end{tabular}

* Binding parameters were recorded for 22 samples of cord serum as described in "Materials and Methods." The slope and intercept are derived by linear least-squares fit with peroxidase as the dependent and fluorescence quenching as the independent variable. The correlation coefficient (Pearson) is denoted $r$ and the significance level is $P$.

$\dagger \mathrm{RBBC}$, reserve bilirubin binding capacity.

clinician in favor of exchange transfusion. From Figure 1, it is apparent that by the peroxidase method three samples (two from a single baby) were in the presumed danger range. The values shown were derived from a Scatchard plot; if the original unbound bilirubin values (before titration) are used instead, three additional values would be greater than $20 \mathrm{nM}$. No values above $20 \mathrm{nM}$ were recorded with the fluorescence quenching method. Exchange transfusion was performed based on "conventional"
Table 2. Comparison of mean values of binding parameters between high risk newborns and healthy term newborns (cord samples)*

\begin{tabular}{lccc}
\hline \multicolumn{1}{c}{ Parameter } & $\begin{array}{c}\text { High risk } \\
\text { mean } \pm \mathrm{SD}\end{array}$ & $\begin{array}{c}\text { Healthy } \\
\text { mean } \pm \mathrm{SD}\end{array}$ & $P$ \\
\hline $\mathrm{K}_{\mathrm{d}}$ (fluorescence quench- & $1.22 \pm 0.65$ & $1.02 \pm 0.19$ & 0.45 \\
$\quad \mathrm{ing}) \times 10^{-8} \mathrm{M}$ & & & \\
$\mathrm{K}_{\mathrm{d}}$ (peroxidase) $\times 10^{-8} \mathrm{M}$ & $1.22 \pm 0.73$ & $1.34 \pm 0.27$ & 0.15 \\
$\mathrm{n}$ (fluorescence quenching) & $1.00 \pm 0.13$ & $1.03 \pm 0.11$ & 0.25 \\
$\mathrm{n}$ (peroxidase) & $1.03 \pm 0.17$ & $1.07 \pm 0.07$ & 0.33 \\
$\nu$ & $0.41 \pm 0.18$ & $0.05 \pm 0.04$ & $<0.001 \dagger$ \\
$\mathrm{RBBC}$ (fluorescence & $0.59 \pm 0.15$ & $0.98 \pm 0.12$ & $<0.001 \dagger$ \\
$\quad$ quenching) & $0.61 \pm 0.18$ & $1.01 \pm 0.08$ & $<0.001 \dagger$ \\
$\begin{array}{l}\mathrm{RBBC} \text { (peroxidase) } \\
\text { Unbound bilirubin (fluo- } \\
\quad \text { rescence quenching) }\end{array}$ & $0.79 \pm 0.43$ & $0.08 \pm 0.03$ & $<0.001 \dagger$ \\
$\begin{array}{l}\text { Unbound bilirubin (perox- } \\
\text { idase) }\end{array}$ & $0.84 \pm 0.61$ & $0.06 \pm 0.02$ & $<0.001 \dagger$ \\
\hline
\end{tabular}

* Binding measurements were as described in "Materials and Methods." Mean values were compared via two-tailed $t$ test, with significance levels $P$ as shown above. Daggers denote significant differences between the two groups. It is apparent that the differences in reserve bilirubin binding capacity $(\mathrm{RBBC})$ and unbound bilirubin are trivial consequences of the differences in $\nu$, which is in turn primarily a consequence of differences in total bilirubin concentration between groups. 
Table 3. Dependence of binding parameters on birth weight and estimated gestational age*

\begin{tabular}{|c|c|c|c|c|}
\hline \multirow[b]{2}{*}{ Parameter } & \multicolumn{2}{|c|}{ Birth weight } & \multicolumn{2}{|c|}{$\begin{array}{l}\text { Estimated gesta- } \\
\text { tional age }\end{array}$} \\
\hline & $r$ & $P$ & $r$ & $P$ \\
\hline$\nu$ & -0.04 & 0.37 & 0.02 & 0.44 \\
\hline $\begin{array}{l}\mathrm{K}_{\mathrm{d}} \text { (fluorescence quench- } \\
\text { ing) }\end{array}$ & 0.06 & 0.33 & 0.01 & 0.47 \\
\hline$K_{d}$ (peroxidase) & -0.15 & 0.15 & -0.12 & 0.21 \\
\hline n (fluorescence quenching) & -0.01 & 0.48 & 0.05 & 0.34 \\
\hline n (peroxidase) & 0.12 & 0.20 & 0.08 & 0.33 \\
\hline $\begin{array}{l}\text { RBBC (fluorescence } \\
\text { quenching) }\end{array}$ & 0.05 & 0.36 & 0.02 & 0.44 \\
\hline RBBC (peroxidase) & 0.21 & 0.08 & 0.09 & 0.28 \\
\hline $\begin{array}{l}\text { Unbound bilirubin (fluo- } \\
\text { rescence quenching) }\end{array}$ & -0.13 & 0.17 & -0.15 & 0.13 \\
\hline $\begin{array}{l}\text { Unbound bilirubin (perox- } \\
\text { idase) }\end{array}$ & -0.32 & $0.01 \dagger$ & -0.28 & $0.03 \dagger$ \\
\hline
\end{tabular}

* Binding parameters were recorded as described in "Materials and Methods." Gestational age was estimated by pediatric house officers in the standard fashion. The only parameter showing a significant association with birth weight and gestational age was the unbound bilirubin by the peroxidase method (daggers). RBBC, reserve bilirubin binding capacity.

Table 4. Association of binding parameters with Intralipid infusion (mean $\pm S D)^{*}$

\begin{tabular}{lccc}
\hline \multicolumn{1}{c}{ Parameter } & + Intralipid & - Intralipid & $P$ \\
\hline FA/SA & $2.9 \pm 0.4$ & $1.6 \pm 0.7$ & $<0.001 \dagger$ \\
$\mathrm{K}_{d}$ (fluorescence quench- & $2.3 \pm 1.3$ & $1.1 \pm 0.4$ & $<0.001 \dagger$ \\
$\quad$ ing) $\times 10^{-8} \mathrm{M}$ & & & \\
$\mathrm{K}_{\mathrm{d}}$ (peroxidase) $\times 10^{-8} \mathrm{M}$ & $1.8 \pm 0.7$ & $1.1 \pm 0.6$ & 0.07 \\
n (fluorescence quenching) & $0.94 \pm 0.06$ & $1.00 \pm 0.13$ & 0.18 \\
n (peroxidase) & $0.93 \pm 0.11$ & $1.04 \pm 0.17$ & 0.17 \\
\hline
\end{tabular}

* Eight infants received Intralipid according to standard nursery indications and protocol $\left(1-2 \mathrm{~g} \cdot \mathrm{kg}^{-1} \cdot \mathrm{d}^{-1}\right) ; 52$ samples were obtained from infants not receiving Intralipid. Differences were analyzed via two-tailed $t$ test; daggers denote cases for which there was a significant difference between the two groups. Because these groups were not prospectively randomized, readers should be cautious in assigning causal interpretations to these differences. FA/SA, fatty acid/serum albumin molar ratio.

clinical criteria for 10 of the newborns. The two babies with high unbound bilirubin levels by the peroxidase method received exchange transfusion.

Intravenous fat emulsion administration was associated with higher values of $\mathrm{K}_{\mathrm{d}}$ and no differences in the value of $\mathbf{n}$ by either method (Table 4). Newborns receiving fat emulsion had higher FA/SA molar ratios (Table 4). The fluorescence quenching method showed a weak relationship between FA/SA molar ratio and $\mathrm{K}_{\mathrm{d}}(r=0.33, P=0.012)$ and $\mathrm{n}(r=-0.35, P=0.008)$, but the peroxidase method did not $(r=0.06, P=0.37$ and $r=$ $-0.08, P=0.30$, respectively. Because treatment with intravenous lipid was not prospectively randomized, readers must be cautious in making causal inferences regarding these differences.

In an effort to understand the effects of lipids on the fluorescence quenching assay, we examined the effects of fatty acids on bilirubin binding to albumin (Fig. 2). The first two fatty acids bound to albumin cause essentially no displacement of bilirubin at low or high values of the molar ratio of total bilirubin:human serum albumin. At high values of the total bilirubin/HSA molar ratio there is slight displacement of bilirubin by the third fatty acid, and the fourth fatty acid displaces substantial amounts of bilirubin. These are in qualitative agreement with previous measurements $(3,10,32,47)$. For this albumin sample, the association constant in the absence of fatty acids was $6.2 \times 10^{7} \mathrm{M}^{-1}\left(\mathrm{~K}_{\mathrm{d}}=\right.$ $\left.1.6 \times 10^{-8} \mathrm{M}\right)$. When the FA/SA molar ratios were 4 and 5 , the apparent association constants decreased $\left(\mathrm{K}_{\mathrm{d}}\right.$ increased) to $5.8 \times$ $10^{6} \mathrm{M}^{-1}\left(\mathrm{~K}_{\mathrm{d}}=1.7 \times 10^{-7} \mathrm{M}\right)$ and $2.2 \times 10^{6} \mathrm{M}^{-1}\left(\mathrm{~K}_{\mathrm{d}}=4.4 \times\right.$ $10^{-7} \mathrm{M}$ ), respectively. The value of $n$ was 1.02 in the absence of fatty acids and 0.99 and 1.00 with FA/SA molar ratios of 4 and 5 , respectively. Displacement was also measured using bilirubin absorption spectral shifts, and virtually identical results were obtained. These data imply that methods which measure RBBC or $\mathrm{n}$ alone would not sense this risk of displacement of bilirubin by fatty acids.

In our series of newborns, none had FA/SA molar ratios greater than 3.4. All four babies who had FA/SA molar ratios between 3.0 and 3.4 were receiving intravenous fat emulsion and had low values of the total bilirubin/HSA molar ratio.

\section{DISCUSSION}

The fluorescence quenching method, both as originally described and with the modifications described in "Materials and

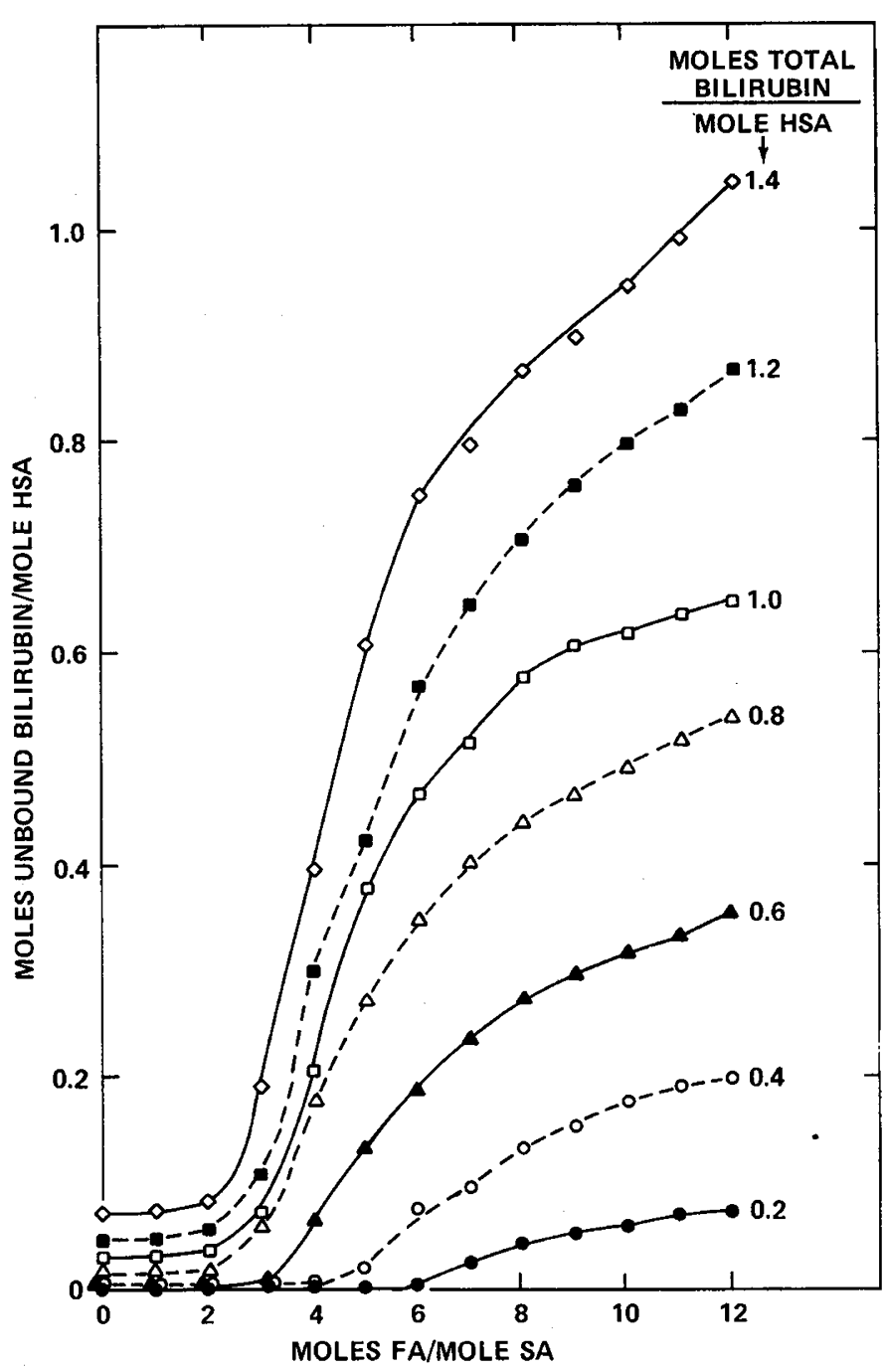

Fig. 2. Displacement of bilirubin from albumin by linolenic acid. The method of fluorescence quenching was used to determine the degree of displacement of bilirubin at various molar ratios of bilirubin and linolenic acid to albumin. For these experiments, the albumin concentration was $2.5 \times 10^{-6} \mathrm{M}$. Spectroscopic methods were as described in "Materials and Methods" and references $(30,39)$. It is apparent that there is very little displacement of bilirubin by the first two fatty acids, and that there is substantial displacement by the fourth and fifth fatty acids. 
Methods," has several potential drawbacks. The method requires a fluorimeter of reasonable quality, preferably with emission and excitation monochromaters and microcuvettes. The measurement is cumbersome, as a great number of additions of bilirubin to the samples is required to achieve a binding curve of sufficient precision. The calculations required to derive the binding parameters are somewhat complex, particularly if detailed inner filter corrections are used. Nevertheless, using the modified procedure described here, we found the fluorescence quenching method to have good reproducibility, in contrast to the results reported by Wells $e t$ al. (44), using the method as described originally (25). The method is least reliable for use at higher values of $\nu$ because it becomes difficult or impossible to determine the initial slope of the quenching curve. Measurement under these conditions, or using a single dilution for both the initial slope and for determination of unbound bilirubin can frequently lead to an underestimation of $\mathrm{K}_{\mathrm{d}}$, which results in an underestimation of the unbound bilirubin and the presumed risk of kernicterus. The great dilution required for assay may lead to an underestimation of displacement due to weakly bound ligands; in this respect, the peroxidase, Sephadex, and fluorescence enhancement methods may be at an advantage. The problem of dilution for fluorescence quenching could potentially be overcome by use of front-face optics, as used in the fluorescence enhancement method. Finally, ligands which quench tryptophan fluorescence or alter the intrinsic quantum yield of tryptophan in albumin (such as furosemide) could produce artifacts in the bilirubin quenching curve (Berde, C. B., unpublished calculations).

The peroxidase assay is based on the assumptions that 1 ) the rate of dissociation of bilirubin from albumin is not rate-limiting in the peroxidase reaction, 2) the loss of optical density at 460 $\mathrm{nm}$ is due only to irreversible oxidation of bilirubin, and 3) there are no significant competing substrates for the peroxidation reaction. Ahlfors (1) has observed that at least the first of these assumptions is not always valid for clinical specimens assayed as originally described; this observation may require performance of the assay at lower dilutions and lower peroxidase concentrations, particularly for samples having low total bilirubin levels. This method does allow measurement of apparent unbound bilirubin concentration without a titration or Scatchard analysis, which may be advantageous for specimens having high values for $\nu$; however, estimation of $\mathrm{K}_{\mathrm{d}}$ and $\mathrm{n}$ for these specimens may not be reliable (48)

Values of $K_{d}, n, R B B C$, and unbound bilirubin determined by these two methods for high-risk infants and on cord serum from normal term infants were significantly correlated (Fig. 1 and Table 2), but there was substantial dispersion of the individual values. In addition, we report slight, but significant dependence of unbound bilirubin on birth weight or gestational age by the peroxidase method (but not by fluorescence quenching), and weak relationships between $K_{d}$ or $n$ and FA/SA molar ratio by fluorescence quenching (but not by the peroxidase method). Because the coefficients of variation for binding parameters by either method are small (see "Materials and Methods" and reference 19), the discrepancies between methods cannot all be due to sampling error alone. The causes of these discrepancies are not apparent. Because both of these procedures are based upon sound physicochemical principles, it is impossible to determine from our data which procedure is in error, or if both are occasionally misleading. The good correlation between the peroxidase method and fluorescence enhancement method reported by Wells et al. (46) suggests, but does not prove, that the discrepancies we report result from errors in measurement by the fluorescence quenching method. Unless a new techniquewhich is unarguably acceptable as a "gold standard"-becomes available for performing these measurements, it will only be possible to determine which methods give "correct" results by performing very careful comparative studies: applying a variety of methods that are all based on sound physicochemical principles to a large number of clinical samples. Good agreement among several assays might then allow other (presumably erroneous) methods to be discarded.

We studied three samples from two babies in which the unbound bilirubin was greater than $20 \mathrm{nM}$ by the peroxidase method, but not by the fluorescence quenching method. It is not clear whether this result is due to overestimation of unbound bilirubin by the peroxidase method or to underestimation by fluorescence quenching. This finding does suggest that results of studies that attempt to correlate risk for bilirubin toxicity with unbound bilirubin levels by one method may not be applicable if unbound bilirubin is measured using another technique.

Our in vitro studies regarding displacement of bilirubin from albumin by fatty acids indicate that displacement occurs when the total bilirubin:albumin mole ratio exceeds 0.6 and the fatty acid:albumin ratio exceeds 3 , but does not become significant at low ratios of total bilirubin:albumin until the fatty acid:albumin mole ratio substantially exceeds 4 . We did not observe any fatty acid:albumin mole ratios in this very high range in our clinical samples, including those from eight babies who were receiving intravenous fat emulsion. These results provide reassurance regarding the safety of intravenous fat emulsion in infants with low total serum bilirubin levels.

The search for methods for measuring bilirubin binding in plasma has been spurred by the observation that kernicterus can occur at relatively low values of $\nu$, by laboratory studies of the effects of displacing ligands, and by clinical experience with the effects of sulfonamide administration on the incidence of kernicterus. As noted by Levine (24) there is little evidence to support, and a number of observations and theoretic considerations which should discourage, the use of measured unbound bilirubin levels in plasma to make inferences about brain bilirubin levels, the susceptibility of the brain to bilirubin toxicity, or the contribution of other factors to the pathogenesis of kernicterus. For a measurement of bilirubin binding parameters to be clinically useful, it must not only be accurate and reliable in measuring those parameters, but those parameters must also be reliable predictors of disease, or specifically, in this case, of neurotoxicity. Although the data presented here addresses only the first of these requirements, we would caution the reader that data addressing the second requirement leave much to be desired. Although Cashore and $\mathrm{Oh}$ (12) found an association of low bilirubin binding capacities and affinities and increased unbound bilirubin levels with gross bilirubin staining of the brains of low birth weight infants, they also noted that there was overlap of the values for the groups of infants with and without staining. Ritter et al. (37), using more strict histopathologic criteria for kernicterus, found no association between unbound bilirubin levels and kernicterus, and also noted overlap of the unbound bilirubin levels for groups of infants with and without kernicterus. But, in reviewing this data, Wennberg and Ahlfors (46) found that the combination of unbound bilirubin levels and serum $\mathrm{pH}$ resulted in a much better correlation with risk for kernicterus. Furthermore, Levine et al. (26) presented evidence which implicates opening of the bloodbrain barrier in the pathogenesis of gross staining of the brain by bilirubin, and Turkel et al. (43) pointed out that gross bilirubin staining of the neonatal brain is often not associated with histopathologic changes indicative of bilirubin toxicity. These studies indicate the need for further studies to carefully delineate the relationships between serum unbound bilirubin levels, brain bilirubin levels, physiologic and pathologic signs of bilirubininduced neurocytotoxicity, and grossly visible staining of the brain. Until these issues have been clarified, we cannot support "widespread introduction of bilirubin binding assays into management of neonatal hyperbilirubinemia," as has been suggested elsewhere (45).

The fluorescence quenching and peroxidase methods are valuable techniques for use in studies of bilirubin metabolism, distribution, and toxicity. The most firmly established use of tests of bilirubin binding is their use in vitro to screen drugs for their ability to displace bilirubin from albumin. Our observa- 
tions, and the experiences of others $(12,24,37)$, however, suggest that routine application of these tests as the primary criterion for therapeutic intervention (e.g., exchange transfusion) is premature. As noted above, a detailed understanding of the physical chemistry of each assay may be required to allow adjustments in technique or even to assess the validity of application in specific clinical situations. An improved understanding of the differences in results by the methods reported here, and recognition of other factors important in the pathogenesis of kernicterus may allow a more selective application of these techniques and enhance the predictive value of their results. Finally, the clinical value of these techniques will be confirmed only by a careful clinical assessment of the specificity and sensitivity of the methods, and by selection of criteria for intervention that ensure that any decrease in the morbidity of hyperbilirubinemia is not exceeded by increased morbidity of treatment.

\section{REFERENCES AND NOTES}

1. Ahlfors, C. E.: Effect of serum dilution on apparent unbound bilirubin concentration as measured by the peroxidase method. Clin. Chem., 27: 692 (1981).

2. Ainbender, E., Brown, E. G., Lee, Y. J., and Sweet, A. Y.: A paper chromatoelectrophoresis method for the determination of bilirubin binding to serum protein. Pediatr. Res., 11: 808 (1977).

3. Andrew, G., Chan, G., and Schiff, D.: Lipid metabolism in the neonate II. The effect of Intralipid on bilirubin binding in vitro and in vivo. J. Pediatr., 88: 279 (1976).

4. Athanassiadis, S., Chopra, D. R., Fisher, M. A., and McKenna, J.: An electrophoretic method for detection of unbound bilirubin and reserve bilirubin binding capacity in serum of newborns. J. Lab. Clin. Med., 83: 968 (1974).

5. Berde, C. B., Hudson, B. S., Simoni, R. D., and Sklar, L. A.: Human serum albumin. Spectroscopic studies of binding and proximity relationships for fatty acids and bilirubin. J. Biol. Chem., 254: 391 (1979).

6. Berde, C. B., Kerner, J. A., and Johnson, J. D.: Use of the conjugated polyene fatty acid parinaric acid in assaying fatty acids in serum or plasma. Clin. Chem., 26: 1173 (1980).

7. Bratlid, D.: Reserve albumin binding capacity, salicylate saturation index, and red cell binding of bilirubin in neonatal jaundice. Arch. Dis. Child, 48: 393 (1973).

8. Brodersen, R.: Bilirubin transport in the newborn infant, reviewed with relation to kernicterus. J. Pediatr., 96: 349 (1980).

9. Brodersen, R.: Free bilirubin in blood plasma of the newborn. Effects of albumin, fatty acids, $\mathrm{pH}$, displacing drugs and phototherapy. Appendix: a provisional survey of the bilirubin-displacing effect of 150 drugs, in Stern L, editor: Intensive care of the newborn. Vol. 2, p. 381 (Masson, New York, NY, 1978).

10. Brodersen, R. and Funding, L.: Binding of bilirubin and long-chain fatty acids to human serum albumin with general remarks on displacement of firmly bound ligands. Scand. J. Clin. Lab. Invest., 37: 257 (1977).

11. Brown, A. K., Eisinger, J., Blumberg, W. E., Flores, J., Boyle, G., and Lamola, A. A.: A rapid fluorimetric method for determining bilirubin levels and binding in the blood of neonates: comparisons with a diazo method and with 2-(4'-hydroxybenzene) azobenzoic acid dye binding. Pediatrics, 65: 767 (1980).

12. Cashore, W. J. and Oh, W.: Unbound bilirubin and kernicterus in low-birth weight infants. Pediatrics, 69: 481 (1982).

13. Cashore, W. J., Oh, W., Blumberg, W. E., Eisinger, J., and Lamola, A. A. Rapid fluorometric assay of bilirubin and bilirubin binding capacity in blood of jaundiced neonates: comparisons with other methods. Pediatrics, 66: 411 (1980).

14. Chen, R. F.: In: A. A. Thaer and M. Sernetz: Fluorescence Techniques in Cell Biology. p. 273. (Springer-Verlag, New York, 1972).

15. Chen, R. F.: Removal of fatty acids from serum albumin by charcoal treatment. J. Biol. Chem., 242: 173 (1967).

16. Doumas, B. T., Watson, W. A., and Biggs, H. G.: Albumin standards and the measurement of serum albumin with bromcresol green. Clin. Chim. Acta, 31:87 (1971).

17. Dupont, C. and Sarkozy, E.: A simplified micromethod for assay of reserve bilirubin binding capacity. J. Lab. Clin. Med., 89: 439 (1977).

18. Fisher, P. F. and Korn, D.: DNA polymerase-alpha. Purification and structural characterization of the near homogenous enzyme from human $\mathrm{KB}$ cells. $\mathrm{J}$. Biol. Chem., 252: 6528 (1977).

19. Griffiths, W. C., Diamond, I., and Dextraze, P.: The albumin binding of unconjugated bilirubin in serum. Clin. Biochem., 8: 254 (1975).

20. Harris, R. C., Lucey, J. F., and MacLean, J. R.: Kernicterus in premature infants associated with low concentrations of bilirubin in the plasma. Pediatrics, 21: 875 (1958).

21. Jacobsen, J. and Wennberg, R. P.: Determination of unbound bilirubin in the serum of newborns. Clin. Chem., 20: 783 (1974).

22. Kaufmann, N. A., Kapitulnik, J., and Blondheim, S. H.: The absorption of bilirubin by Sephadex and its relationship to the criteria for exchange transfusion. Pediatrics, 44: 543 (1969).

23. Lee, K., Gartner, L. M., and Zarafu, I.: Fluorescent dye method for determination of the bilirubin-binding capacity of serum albumin. J. Pediatr., 86 . 280 (1975).
24. Levine, R. L.: Bilirubin: worked out years ago? Pediatrics, $64: 380$ (1979).

25. Levine, R. L.: Fluorescence-quenching studies of the binding of bilirubin to albumin. Clin. Chem., 23: 2292 (1977).

26. Levine, R. L., Fredericks, W. R., and Rapoport, S. I.: Entry of bilirubin into the brain due to opening of the blood-brain barrier. Pediatrics, 69: 255 (1982).

27. Martinek, R. G.: Improved micromethod for determination of serum bilirubin. Clin. Chim. Acta, 13: 161 (1966)

28. McCluskey, S. B., Storey, G. N. B., Brown, G. K., More, D. G., and O'Sullivan, W. J.: Fluorometric determination of "albumin-titratable bilirubin" in the jaundiced neonate. Clin. Chem., 21: 1638 (1975).

29. Morrisett, J. D., Pownall, H. J., and Gotto, A. M.: Bovine serum albumin. Study of the fatty acid and steroid binding sites using spin-labelled lipids. J. Biol. Chem., 250: 2487 (1975).

30. Odell, G. B.: The dissociation of bilirubin from albumin and its clinical implications. J. Pediatr., 55: 268 (1959).

31. Odell, G. B., Cohen, S. N., and Kelly, P. C.: Studies on kernicterus II. The determination of the saturation of serum albumin with bilirubin. J. Pediatr., 74: 214 (1969).

32. Odell, G. B., Cukier, J. O., Ostrea, E. M. Jr., Maglalang, A. C., and Poland, R. L.: The influence of fatty acids on the binding of bilirubin to albumin. $\mathrm{J}$. Lab. Clin. Med., 89: 295 (1977).

33. Ostrow, J. D., Hammaker, L., and Schmid, R.: The preparation of crystalline bilirubin-C ${ }^{14}$. J. Clin. Invest., 40: 1442 (1961).

34. Parsons, D. L.: Analysis of fluorescence quenching data by the method of Levine. Clin. Chem., 26: 1869 (1980).

35. Porter, E. G. and Waters, W. J.: A rapid micromethod for measuring the reserve albumin binding capacity in serum from newborn infants with hyperbilirubinemia. J. Lab. Clin. Med., 67: 660 (1966).

36. Rasmussen, L. F., Ahlfors, C. E., and Wennberg, R. P.: The effect of paraben preservative on albumin binding of bilirubin. J. Pediatr., 89: 475 (1976).

37. Ritter, D. A., Kenny, J. D., Norton, H. J., and Rudolph, A. J.: A prospective study of free bilirubin and other risk factors in the development of kernicterus in premature infants. Pediatrics, 69: 260 (1982).

38. Scatchard, G.: The attractions of proteins for small molecules and ions. Ann. N.Y. Acad. Sci., 51: 660 (1949).

39. Silverman, W. A., Andersen, D. H., Blanc, W. A., and Crozier, D. N.: A difference in mortality rate and incidence of kernicterus among premature infants allotted to two prophylactic antibacterial regimens. Pediatrics, 18 . 614 (1956).

40. Starinsky, R. and Shafrir, E.: Displacement of albumin bound bilirubin by free fatty acids: implications for neonatal hyperbilirubinemia. Clin. Chim. Acta 24: 311 (1970).

41. Stern, L.: Drug interactions-Part II: Drugs, the newborn infant, and the binding of bilirubin to albumin. Pediatrics, 49: 916 (1972).

42. Thiessen, H., Jacobsen, J., and Brodersen, R.: Displacement of albumin bound bilirubin by fatty acids. Acta Paediatr. Scand., 61: 285 (1972).

43. Turkel, S. B., Miller, C. A., Guttenberg, M. E., Moynes, D. R., and Hodgman, J. E.: A clinical pathologic reappraisal of kernicterus. Pediatrics, 69: 267 (1982).

44. Wells, R., Drew J. H., and Hammond, K. B.: Bilirubin binding capacity and free bilirubin concentration: fluorescence quenching compared with peroxidase oxidation and Sephadex column elution techniques. Clin. Chim. Acta, 116: 69 (1981).

45. Wells, R., Hammond, K., Lamola, A. A., and Blumberg, W. E.: Relationships of bilirubin binding parameters. Clin. Chem. 28: 432 (1982).

46. Wennberg, R. P., and Ahlfors, C. E.: Letter to the editor. Pediatrics, 70: 658 (1982).

47. Wennberg, R. P., Rasmussen, L. F., and Ahlfors, C. E.: Displacement of bilirubin from human albumin by three diuretics. J. Pediatr., 90: 647 (1977).

48. Wennberg, R. P., Rasmussen, L. F., Ahlfors, C. E., and Valaes, T.: Mechanized determination of the apparent unbound unconjugated bilirubin concentration in serum. Clin. Chem., 25: 1444 (1979).

49. Woolley, P. V. III, and Hunter, M. J.: Binding and circular dichroism data on bilirubin-albumin in the presence of oleate and salicylate. Arch. Biochem. Biophys., 140: 197 (1970).

50. The present address of C.B.B. is the Department of Anesthesia, Massachusetts General Hospital, Boston, Massachusetts.

51. The present address of J.D.J. is the Department of Pediatrics, University of New Mexico Medical Center, Albuquerque, New Mexico.

52. The authors are indebted to Drs. Philip Sunshine, Rolf Brodersen, and Angelo Lamola for encouragement and helpful discussions, to Drs. Robert Simoni and Bruce Hudson for laboratory space and support, and to the nurses and housestaff at the Stanford and University of California nurseries for their cooperation in carrying out this study.

53. This paper was presented in part at the meeting of the Western Society for Pediatric Research in February of 1979 in Carmel, California.

54. Requests for reprints should be addressed to: Dr. John A. Kerner, Stanford University School of Medicine, Dept. of Pediatrics, S222, Stanford, CA 94305.

55. C.B. was supported by USPHS Grant 01822 as a participant in the Medical Scientist Training Program. At the time of these studies, J.D.J. was an Investigator, Howard Hughes Medical Institute. J.A.K. was supported by $\mathrm{NIH}$ training grant HD 00049-17. These studies were supported in part by a grant (RR-81) from the General Clinical Research Center of the Division of Research Resources, NIH, and by NIH grants GM 18539 and GM 21149.

56. Received for publication January $20,1983$.

57. Accepted for publication July 12, 1983. 